\title{
Modulo-Variable Expansion Sensitive Scheduling
}

\author{
Madhavi Gopal Valluri $\nmid$ and R. Govindarajan $\dagger \ddagger$ \\ $\dagger$ Supercomputer Education and Research Centre \\ $\ddagger$ Department of Computer Science and Automation \\ Indian Institute of Science, Bangalore 560 012, India \\ madhavi@rishi.serc.iisc.ernet.in \\ govind@ $\{$ serc, csa $\}$.iisc.ernet. in
}

\begin{abstract}
Modulo scheduling is an aggressive scheduling technique for loops that exploits instruction-level parallelism by overlapping successive iterations of the loop. Due to the nature of modulo scheduling, the lifetime of a variable can overlap with a subsequent definition of itself. To handle such overlapping lifetimes, modulovariable expansion (MVE) is used, wherein the constructed schedule is unrolled a number of times.

In this paper we propose a technique to improve the constructed schedule while performing MVE. In our approach, we unroll the data dependence graph of the original loop and re-schedule it with a MVE-sensitive scheduler. Such an approach is expected to result in better initiation rates as compared to the traditional approach. We have implemented our approach and evaluated its performance on a large number of scientific benchmark kernels.
\end{abstract}

\section{Introduction}

Modulo scheduling or software pipelining is an aggressive instruction scheduling technique for exploiting instruction-level parallelism in loops $[6,11]$. In this scheduling method, successive iterations of the loop are initiated at a constant interval, known as the initiation interval (II) of the schedule. The constructed schedule must obey the resource constraints of the machine as well as the dependency constraints of the loop. Several methods have been proposed to perform software pipelining $[1,2,3,5,6,9,8,11,10,13]$.

Due to the nature of modulo scheduling, the lifetime of a variable can overlap with a subsequent definition of itself. To handle such overlapping lifetimes, some form of register renaming has to be provided to ensure that successive definitions of a variable use distinct registers. Some architectures provide hardware support in the form of rotating register files [14]. When no hardware support is available, the problem of overlapping lifetimes can be solved by using Modulo-Variable Expansion (MVE), wherein the kernel is unrolled and multiple definitions of a variable are renamed at compile time [6]. In the traditional approach, when MVE is required, the constructed schedule is unrolled a number of times. The disadvantage of this is that the final schedule obtained by unrolling the schedule will have an II which is an exact multiple of the II of the original loop. Even if there exists a valid schedule at an II less than the exact multiple, the traditional method does not exploit this possibility. By a valid schedule we mean a schedule that obeys the resource and data dependency constraints, and does not need further MVE.

We propose a technique that attempts to find such a schedule by unrolling the data dependence graph (DDG) of the loop and re-scheduling it with a MVEsensitive scheduler. By unrolling the DDG, we also overcome the limitation that the II of the original loop is restricted to be an integer. If the lower bound on the II computed before scheduling is not an integer, the performance degradation caused by rounding it up to an integer may be reduced by unrolling the loop. Our approach attempts to take advantage of this. The approach proposed in [7] by Lavery and Hwu also attempts to overcome the same limitation by unrolling the loop a number of times. However, they unroll the loop before modulo scheduling. Their final schedule may still require MVE, in which case they perform MVE using the traditional approach.

The proposed MVE-sensitive scheduler was implemented and tested on a large number of standard scientific kernels for three different machine models including the MIPS R8000 processor [4]. We used Huff's Lifetime-Sensitive Modulo Scheduling algorithm as the 
base scheduling method [5]. Our experiments show that a large number of loops (27\% to $50 \%$ of the loops) required MVE. Of these, our experimental analysis revealed potential for improvement in II in about 35\% of the loops. However the improvement in II was observed only in $6 \%$ of the loops. This can be attributed to the possibly high resource requirement of the MVEsensitive scheduler.

The rest of the paper is organized as follows. Section 2 gives the necessary background on modulo scheduling and modulo-variable expansion through a motivating example. Section 3 describes our scheduling technique in detail. Experimental results are reported in Section 4 and Section 5. Section 6 summarizes the conclusions.

\section{Background and motivating example}

In this section, first we present the necessary background on modulo scheduling and modulo-variable expansion through a motivating example. Using the example, subsequently, we motivate our approach to improve the constructed schedule while performing modulo-variable expansion.

\subsection{Modulo Scheduling}

In a modulo scheduled loop the constant interval between the initiations of successive iterations is called Initiation Interval (II) [10]. The II of a software pipelined schedule is governed by both resource constraints and recurrences in the loop. The minimum initiation interval MII is the maximum of Recurrence MII (RecMII), due to inter-iteration dependences in the loop, and Resource MII (ResMII), due to the resource constraints of the architecture [10]. RecMII and ResMII are defined as

$$
\text { RecMII }=\max _{\text {All cycles }} \frac{\text { Length of recurrence cycle }}{\mathrm{D}}
$$

where Length of recurrence cycle is the sum of latencies of all instructions in the cycle and $D$ represents the sum of dependence distances on the arcs in the cycle. Dependence distance or loop-carried dependence on an arc indicates how many iterations later the value produced by an instruction is consumed by its successor.

$$
\text { ResMII }=\max _{r \in \text { set of }} \text { FU types } \frac{N_{r} * d_{r}}{F_{r}}
$$

where $N_{r}$ represents the number of instructions that are executed in function unit (FU) type $\mathrm{r}, d_{r}$ represents the maximum number of cycles during which any stage of FU type $\mathrm{r}$ is used by an instruction, and $F_{r}$ is the number of FUs in type r. Lastly,

$$
\mathrm{II}>\mathrm{MII}=\max (\lceil\mathbf{R e c M I I}\rceil,\lceil\text { ResMII }\rceil) .
$$

Further details on RecMII and ResMII can be found in $[5,6,10,13]$. Consider the data dependence graph

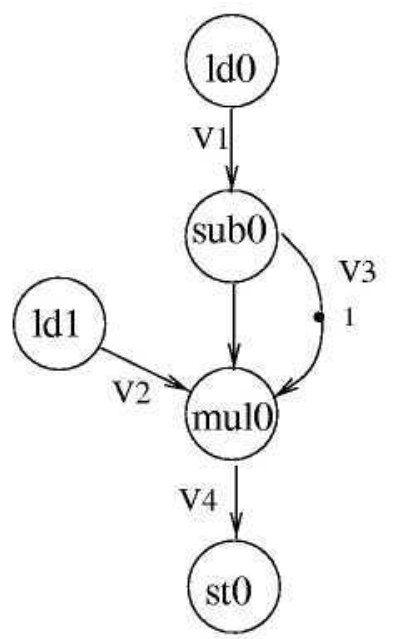

(a)

\begin{tabular}{|l|c|c|c|}
\hline \multicolumn{1}{|c|}{ Func Units } & $\begin{array}{c}\text { Number } \\
\text { of Units }\end{array}$ & Latency & Pipelined \\
\hline Load/Store & 2 & 2 & yes \\
Add/Sub & 1 & 1 & yes \\
Multiply & 1 & 1 & yes \\
\hline
\end{tabular}

(b)

\section{Figure 1. (a) Motivating Example (b) Machine Configuration}

(DDG) shown in Figure 1(a), and a machine configuration as shown in Figure 1(b). Each node in the DDG represents an operation and an arc represents data dependence between operations. Dependence distance is represented by means of a token on the arc. Since there are no recurrence paths, the MII of the DDG is the ResMII, which is 2. The loop has a schedule at MII as shown in Figure 2(a). 

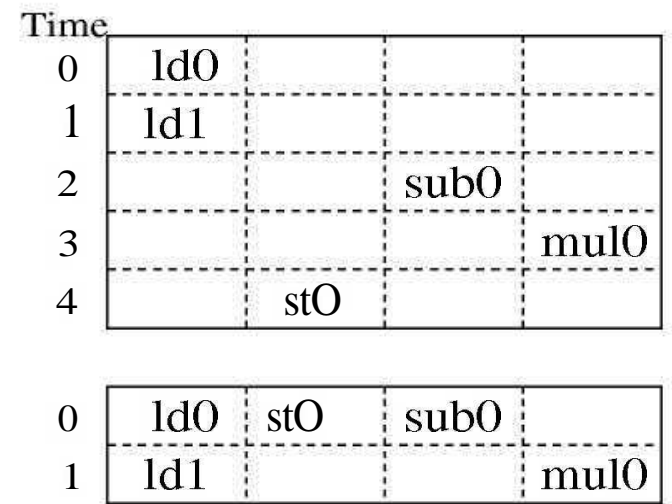

Figure 2. (a) Schedule of Loop shown in Fig.1 (b) Kernel

\subsection{Modulo-Variable Expansion}

A variable defined by an operation needs to be stored and retained in a register until all its successors have used the value. The lifetime of a variable is defined as the duration between the first assignment and its last use. Due to the nature of modulo scheduling, the lifetime of a variable defined in one iteration can overlap with the lifetimes of variables defined in successive iterations. Furthermore, for a variable with a lifetime greater than II, new values are generated before the previous one is used, i.e., the lifetime of a variable can overlap with a subsequent definition of itself. For

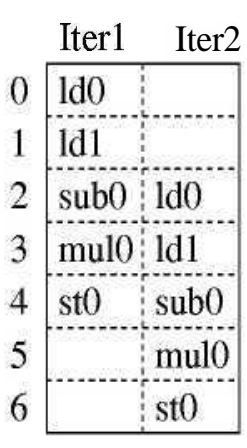

(a)

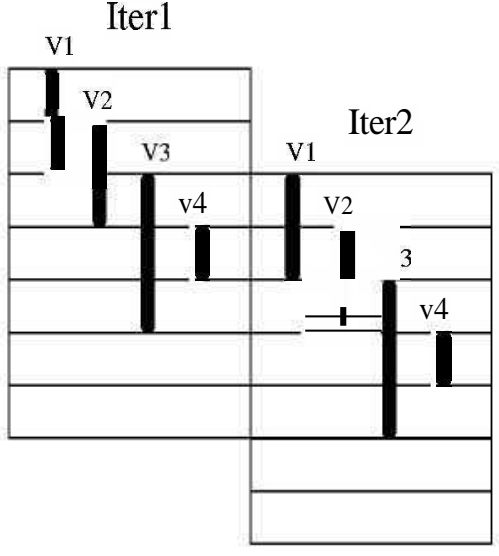

(b)
Figure 3. (a) Schedules of two consecutive iterations and (b) Lifetimes of variables

the motivating example, the lifetimes of all variables for two consecutive iterations of the loop are shown in
Figure 3(b). We see that lifetime of the virtual register V3 begins in cycle 2, i.e., the cycle in which the operation subO starts execution, and ends in cycle 5 which corresponds to the last use, i.e., use by the operation mulO from the next iteration. Thus, the lifetime of V3 has a length of 3 cycles, which is greater than the II of the loop. Hence this lifetime overlaps with the next definition of itself, which occurs in cycle 4 as shown in thefigure.

To handle such overlapping lifetimes, some form of register renaming has to be provided to ensure that successive definitions of the variable use distinct registers. Some architectures provide hardware support in the form of rotating register files [14]. Rotating register files rename different instantiations of a variable during execution time.

When no hardware support is available, the problem of overlapping lifetimes can be solved by using Modulo-Variable Expansion(MVE), wherein the kernel is unrolled and multiple definitions of a variable are renamed at compile time [6].

In the traditional approach, when MVE is required, the constructed schedule is unrolled a number of times. For any loop the required degree of unroll, henceforth referred to as unroll_factor, is determined by the longest lifetime among the variables [12]. Because iterations are initiated every II cycles, unrolLfactor can be calculated as :

$$
\text { Unroll Factor }=\max _{i}\left\lceil\frac{\text { lifetime, }}{\text { II }}\right\rceil
$$

The unrolled schedule has an II which is equal to the II of the original loop multiplied by the unrolLfactor. In our example, the kernel has to be unrolled $\left\lceil\frac{3}{2}\right\rceil=2$ times. In this case, the II of the unrolled schedule is 4 .

\subsection{Our Approach}

In our approach, instead of unrolling the schedule, we unroll the DDG itself and schedule it. The unrolled DDG is shown in Figure 4. Again, since there are no recurrence paths, the MII of the unrolled DDG is the ResMII, which is 3. When the unrolled DDG is scheduled, we see that it gets scheduled at an II of 3 , as opposed to an II of 4 achieved by the traditional approach. The schedule and lifetimes of the unrolled DDG are shown in Figure 5(a) and Figure 5(b) respectively. While scheduling the unrolled DDG, we have to ensure that the schedule obtained from the unrolled DDG will not require further modulo-variable expansion, i.e., the scheduler which schedules the unrolled DDG should be sensitive to modulo-variable expansion. In other words, we should ensure that the lifetime of 


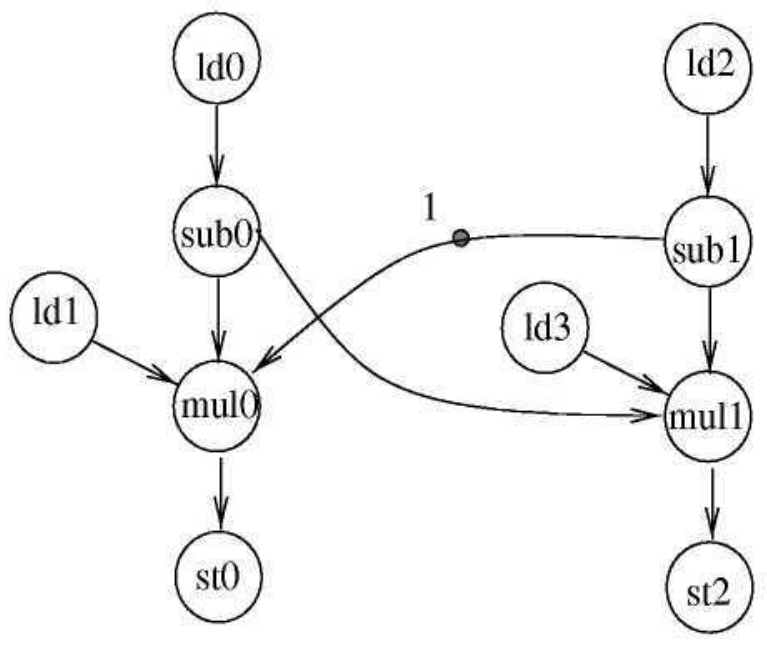

Figure 4. Unrolled DDG

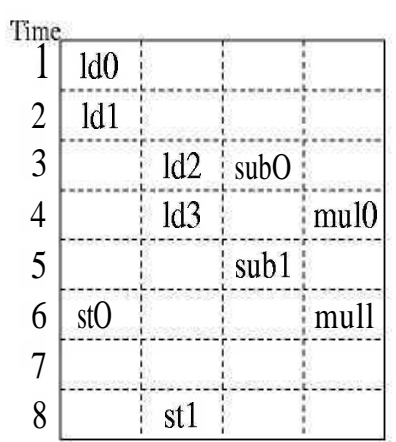

(a)

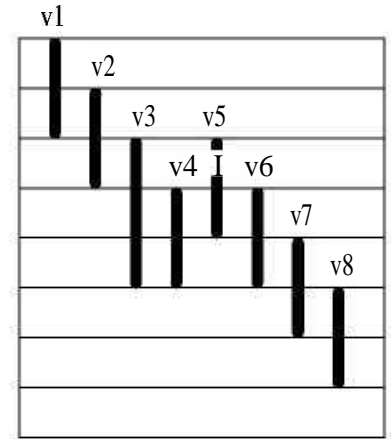

(c)

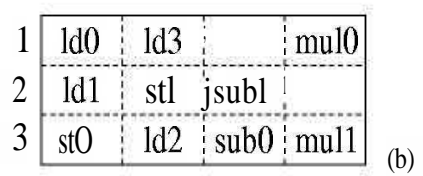

\section{Figure 5. (a) Schedule of unrolled loop (b) Ker- nel (c) Lifetimes}

a virtual register in the final schedule for the unrolled DDG does not overlap with a subsequent definition of itself. In order to ensure this, we derive the necessary conditions in Section 3.3. The following section discusses the scheduling method used in our work.

\section{Scheduling Technique}

In this section we describe our scheduling technique which is sensitive to modulo-variable expansion. The method is based on Huff's Lifetime-Sensitive Modulo Scheduling algorithm [5]. In Section 3.1 basic definitions of a few terms used in Huff's algorithm and in our approach are given. For the sake of completeness we briefly describe Huff's algorithm in Section 3.2. Our approach is described in Section 3.3. Readers familiar with Huff's algorithm could skip Sections 3.1 and 3.2.

\subsection{Definitions}

This subsection defines certain basic terminology .

Definition 3.1 (Mindist) mindist $(i, j)$ is the minimum number of cycles by which operation $i$ must precede operation $j$ in any feasible schedule.

Intuitively, mindist $(\mathrm{i}, \mathrm{j})$ corresponds to the maximum distance in terms of execution latencies of operations along any path from $i$ to $j$.

Definition 3.2 (Estart) Estart is the earliest time step at which an operation can be scheduled such that all its predecessors have completed their execution.

Definition 3.3 (Lstart) Lstart is the latest time step at which an operation can be scheduled such that it completes its execution before its successors can start executing.

In Section 3.2 we explain how Estart and Lstart of an operation can be computed.

Definition 3.4 (Slack) Slack of an operation $i$ is defined as the difference between its Lstart and Estart values. i.e.,

$$
\operatorname{Slack}(i)=\operatorname{Lstart}(i)-\operatorname{Estart}(i)
$$

\subsection{Huff's Algorithm}

Huff's Lifetime-Sensitive Modulo Scheduling algorithm schedules a loop based on the scheduling freedom or slack of an operation. The method attempts to schedule a loop for minimal register pressure as well as minimal II. To aid in the scheduling process, two pseudo-operations are added to the loop body: Start and Stop. Start is the predecessor of each operation and Stop is the successor of every operation. Using these nodes and the mindist relation, the scheduler initializes the Estart and Lstart bounds for each operation to:

$$
\text { Estart }(i)=\text { mindist }(\text { Start }, i)
$$

and

$$
\text { Lstart }(i)=\operatorname{Lstart}(\text { Stop })-\text { mindist }(i, \text { Stop })
$$

Once the initial bounds are set up, the scheduler is ready to place the operations one by one into the partial 
schedule. The algorithm tries to place operations into the partial schedule until either a feasible schedule is found or the heuristics give up after a large number of tries. The algorithm consists of the following basic steps:

- Choose an operation to place in the partial schedule based on a priority function. Huff's algorithm prioritizes operations based on their slacks.

- Search for a valid issue slot within the Estart and Lstart bounds of the operation. An issue slot is valid if placing the operation in that slot does not create any resource conflict in the partial schedule.

- If no conflict-free slot is found, then the operation is forced into the time-slot

$$
\max (\text { Estart, }(\text { PrevScheduleTime }+1) \text { ) }
$$

where PrevScheduleTime is the time at which the operation was scheduled previously. After forcing the operation, all operations in the partial schedule whose resource requirements conflict with the current operation are ejected. Further, if the operation is placed beyond its Lstart, all its successors are also ejected.

- Once the operation has been placed, the modulo reservation tables are updated to indicate the resource usage of the placed operation.

- The Estart and Lstart bounds of all unscheduled operations are updated to reflect the placement of the new operation. Let a new operation $i$ be scheduled in time-step $t i$, then, for each operation $j$ not in the partial schedule the bounds are updated to :

$$
\operatorname{Estart}(j)=\max (\operatorname{Estart}(j), t i+\operatorname{mindist}(i, j))
$$

and

$$
\operatorname{Lstart}(j)=\min (\operatorname{Lstart}(j), t i-\operatorname{mindist}(j, i))
$$

- If the number of operations ejected is beyond some threshold value, then all operations are removed from the partial schedule, II is incremented and all the above steps are repeated.

\subsection{Our Scheduling approach}

Once a valid schedule of the loop is found using Huff's algorithm, the lifetimes of all variables are computed. If any of the variables has a lifetime greater than II, then MVE has to be performed on the loop.
In the traditional approach, when MVE is required, the constructed schedule is unrolled. In our approach we unroll the DDG of the original loop and re-schedule it with a modulo scheduler sensitive to MVE.

In subsequent sections we will use the following notations to differentiate the II and MII of the original loop and the unrolled loop: Let,

$I I_{\text {org }}$ denote the II the original loop.

$M I I_{\text {org }}$ denote the MII of the original loop.

$I I_{\text {unroll }}$ denote the II of the unrolled loop.

$M I I_{\text {unroll }}$ denote the MII of the unrolled loop.

The scheduler which schedules the unrolled loop must adhere to the following conditions :

Condition A The schedule obtained for the unrolled $D D G$ should not require further modulo-variable expansion. Henceforth we refer to this condition as the MVE constraint.

Condition B The schedule obtained for the unrolled DDG should have an II less than $I I_{\text {org }}$ * unroll_factor. This constraint is mainly to ensure that scheduling the unrolled DDG is at least as efficient as unrolling the schedule.

In order to satisfy the Condition A the scheduler must ensure that the lifetime of a variable can never exceed Hunroii and hence will not need MVE. To achieve this, the relations for Estart and Lstart bounds used in Huff's algorithm have to be refined. To see how Estart and Lstart bounds have to be modified, consider the two operations shown in Figure 6(a). Let the opO be scheduled in time-step $t_{0}$ and let op 1 be a direct unscheduled successor of it. The Lstart of opl is first found using Huff's Lstart bounds to satisfy the mindist relation. It is computed depending on the successors of opl (not shown in the figure). Then, in order to ensure that the lifetime of the variable defined by opO does not exceed $I I_{\text {unroll }}$, op1 should be scheduled before $t_{0}+$ Hunroii cycles. Therefore the Lstart of opl is defined as:

$$
\text { Lstart }(\mathrm{opl})=\min \left(\text { Lstart }(\mathrm{op} 1), \mathrm{t}_{0}+I I_{\text {unroll }}\right)
$$

Now, consider the operations shown in Figure 6(b). In this case the dependence distance from opO to opl is greater than zero ( 1 in case of Figure $6(\mathrm{~b}))$. Assume again that opO is scheduled in time-step $t_{0}$. Here, opO produces a value which is used by opl in the next iteration. In other words, opl uses the value defined by opO in cycle $t_{1}+I I_{\text {unroll }}$, where $t_{1}$ is the time-step in which opl is scheduled. Hence, the lifetime of the 


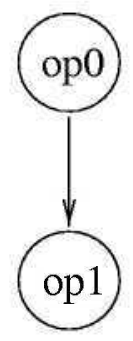

(a)

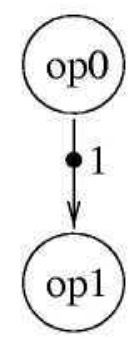

(b)
Figure 6. Lstart Calculation

variable is $t_{1}+I I_{\text {unroll }}-t_{0}$. By the MVE constraint this should be less than $I I_{\text {unroll }}$, i.e,

$$
\mathrm{t}_{1}+I I_{\text {unroll }}-\text { to }<I I_{\text {unroll }}
$$

We see that in this case opl should be scheduled before time-step $t_{0}$.

In general, for any dependence distance $d$, we have to ensure that

$$
\mathrm{t}_{1}+\boldsymbol{d} * I I_{\text {unroll }} \text { - to }<I I_{\text {unroll }}
$$

Therefore, if an operation $i$ is scheduled, for each unscheduled operation $j$ which is a direct successor of operation $i$, the Lstart relation can be written as :

$$
\text { Lstart }(j)=m m\left(\operatorname{Lstart}(j), t_{\mathrm{i}}+(1-d) * I I_{\text {unroll }}\right)
$$

where $t_{i}$ is the time-slot in which operation $i$ is placed.

We see that in order to satisfy the MVE constraint, the Lstart of an operation depends not only on its successors as in Huff's original algorithm, but also depends on its direct predecessors.

Similarly, the Estart relation can be derived as :

Estart $(j)=\max \left(\operatorname{Estart}(j), t_{i}+(d-1) * I I_{\text {unroll }}\right)$

Thus, in order to satisfy Condition A the MVEsensitive scheduler uses the above modified equations for Estart and Lstart calculations.

In order to satisfy Condition B, our scheduler searches for a valid schedule only between $M I I_{\text {unroll }}$ and $I I_{\text {org }}$ * unroll_factor. If a valid schedule is not found in this range, the scheduler outputs the unrolled schedule as in the traditional approach.

Our MVE-sensitive scheduler incorporates the above mentioned modifications in Huff's algorithm and attempts to find a final schedule for the unrolled DDG.

The overall algorithm consists of the following steps:

Step 1 Schedule the original loop using Huff's algorithm.
Step 2 Find the lifetimes of all variables.

Step 3 Find the unroll__actor using relation given in Section 2.2.

Step 4 Unroll the DDG the required number of times.

Step 5 Schedule the unrolled DDG using the MVEsensitive scheduler to obtain a valid schedule that does not require further MVE.

\section{Experimental Setup}

Our modulo scheduling approach, sensitive to modulo-variable expansion, is based on Huff's slack scheduling algorithm [5]. We used Huff's method because it is a fast and efficient method. However the approach presented in this paper can be applied to any modulo scheduling algorithm. The approach was tested for its performance on a set of 1450 benchmarks. These are basic blocks, extracted from a variety of scientific benchmark programs like Linpack, Livermore, NAS, and SPEC. We assumed three machine configurations with complex structural hazards. The first configuration closely resembles the R8000 machine model (Model 1). The other two are hypothetical models.

Model 1 This configuration resembles the R8000 machine model. It consists of eighteen functional units, details of which can be found in [4].

Model 2 This is a simple hypothetical model consisting of two integer add units with unit latency, one integer load unit with latency 3 , one integer store unit with latency 4 , one floating point add unit with latency 4 , one floating point multiply unit with latency 3 , and one divide unit with latency 18 .

Model 3 This model has the same number of functional units as Model 2 but with more complex structural hazards and higher latencies. The integer add, integer load and floating point add units have a latency of 5 , integer store unit has a latency of 4, floating point multiply unit has a latency of 7, and the floating point divide has a latency of 22.

The performance results for all the three machine configurations are presented in the following section.

\section{Results}

The performance results for the three machine models are tabulated in Tables 1 and 2 . 


\begin{tabular}{|l|c|c|c|c|}
\hline Machine Model & $\begin{array}{c}\text { Loops which } \\
\text { required MVE }\end{array}$ & $\begin{array}{c}\text { Loops in which } \\
M I I_{\text {unroll }} \\
I I_{\text {org }} \text { * unrolLfactor }\end{array}$ & $\begin{array}{c}\text { Loops in which } \\
M I I_{\text {unroll }}< \\
I I_{\text {org }} \text { * unr } \text { oll } \text {-factor }\end{array}$ & $\begin{array}{c}\text { Loops scheduled } \\
\text { with an improved } \\
I I_{\text {unroll }}\end{array}$ \\
\hline Model 1 & 388 & 226 & 162 & 27 \\
\hline Model 2 & 502 & 352 & 150 & 29 \\
\hline Model 3 & 718 & 470 & 248 & 42 \\
\hline
\end{tabular}

Table 1. Statistics on Improvement

A total of 1450 loops were run on the scheduler. Out of the 1450 loops about $49.4 \%$ of the loops required MVE in case of Model 3. The number of loops requiring MVE was less (27\% and 35\%) in case of Models 1 and 2. This is possibly because both Models 1 and 2 have simpler resource configurations compared to Model 3. From here on we discuss the results for Model 3 in greater detail while the results for Models 1 and 2 are tabulated.

In case of Model 3, out of the 718 loops that needed MVE, 470 loops (65\%) of the loops had an $M I I_{\text {unroll }}$ equal to $I I_{\text {org }} *$ unroll_factor. This is because these loops got scheduled at their MII and the absolute lower bound on their MII was an integer, hence when unrolled, the unrolled DDG had an MII which was $M I I_{\text {org }}$ * unroll_factor. In these loops our MVEsensitive scheduler would not have been able to give any performance gains. Therefore, our scheduler follows the traditional approach and outputs the unrolled schedule. In the remaining 248 (35\%) loops there was potential for improvement in II. However, we could get schedules at an improved II only in about $42(6 \%)$ of the loops. For the loops in which we did get an improvement in II, the improvement was significant (refer to Table 2). The performance improvement was calculated' as $\frac{I I_{\text {org }} * \text { unroll }}{I I}$ factor-II $-I I_{\text {uroll }}$, where $\Pi_{\text {unroll }}$ was the II obtained by the MVE-sensitive scheduler.

In Model 3, the improvement in II was as high as $37.5 \%$. The average improvement in II was $12 \%$. The performance improvements in Model 1 and 2 were higher. In Model 1 we observed a performance improvement of about $14 \%$ and in Model 2 the average performance improvement was about $23 \%$.

\begin{tabular}{|l|c|c|}
\hline Machine Model & Maximum \% & Average \% \\
\hline Model 1 & 33.3 & 13.8 \\
\hline Model 2 & 60.0 & 23.1 \\
\hline Model 3 & 37.5 & 12.0 \\
\hline
\end{tabular}

Table 2. Improvement in II
The loops which could not be scheduled at an $I I<$ $I I_{\text {org }}$ * unroll_fictor even though there was potential for improvement in them, is due to fact that the MVE constraint requires certain nodes to be placed in certain time slots which aggravates the resource requirements considerably. To empirically establish this we ran all the loops on an infinite resource model, i.e., with no resource constraints. The unrolled loops were scheduled using the MVE-sensitive scheduler. It was observed that all the loops got scheduled at the $M I I_{\text {unroll }}$ of the loops. In this experiment, we used the value of the $M I I_{\text {unroll }}$ for each loop as computed for the target machine model, i.e., with resource constraints. We did not attempt to schedule the loops for II $=M I I_{\text {unroll }}$ of the infinite resource model. This is because our interest here is to find out whether the MVE constraint does really increase the resource requirement, and that it prevents the loops to be scheduled at an II less than IIorg * unroll_factor.

In [7], various unrolling-based optimizations have been proposed to reduce the resource requirements and the height of the critical path of the unrolled loop body. Some of these optimizations include removing the loop exit branches from the unrolled loop body, induction variable elimination etc. Currently none of the optimizations have been included in our implementation. It is important to note that our approach can further benefit from these optimizations, whereas the traditional approach (of unrolling the schedule) cannot.

In our approach, we first compute the schedule for the original loop using Huff's scheduling method, and subsequently we schedule the unrolled loop, if MVE is needed, using the MVE-sensitive scheduler. This requires scheduling to be performed twice. Alternatively, it is possible to apply the MVE-sensitive scheduler on the original loop and obtain a schedule that does not require MVE. However, there are some loops which may inherently require MVE; e.g., this happens if the DDG of the loop consists of forward edges with dependence distance greater than one [8]. In these loops it is not possible to get a schedule that does not require MVE without unrolling it. Hence, we first estimate the min- 
imum unroll_factorusing the mindist relation as:

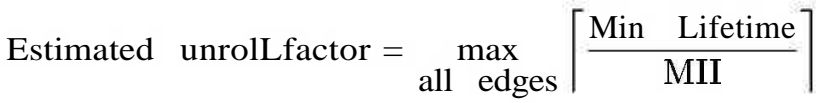

where minimum lifetime is computed using the mindist relation. The loop is then unrolled by the Estimated unrolLfactor and the MVE-sensitive scheduler is applied to it. Hence, this approach eliminates the preliminary scheduling step.

The performance of this approach was measured for machine Model 3 for the 718 loops which needed MVE. The results showed that using this approach only 576 of the 718 loops could be scheduled and out of the 576 loops only in 20 of the loops the II for the original loop was better than the II obtained by using Huff's original approach. In all the remaining loops (556 loops), Huff's method produced schedules with a lower II.

\section{Conclusions}

Modulo-variable expansion is an essential, compiletime renaming technique required for certain modulo scheduled loops. In this paper we propose a technique to improve the constructed schedule while performing modulo-variable expansion. We improve, in terms of II, the schedule by unrolling the data dependence graph (DDG) of the loop and re-scheduling it rather than simply unrolling the schedule as in the traditional approach. We have derived the conditions that the scheduler which schedules the unrolled DDG must adhere to. These conditions ensure that our MVEsensitive scheduler which schedules the unrolled DDG is at least as efficient as the traditional approach.

We have tested the approach for 1450 standard benchmarks and for three machine configurations. We have shown that the MVE-sensitive scheduler finds schedules at a lower II for about $6 \%$ of the loops. In those loops, there is an average improvement in II from $12 \%$ to $23 \%$.

\section{References}

[1] James C. Dehnert and Ross A. Towle. Compiling for Cydra 5. Jl. of Supercomputing, 7:181-227, May 1993.

[2] Alexandre E. Eichenberger and Edward S. Davidson. Stage scheduling: A technique to reduce the register requirements of a modulo schedule. In Proc. of the 28th Ann. Intl. Symp. on Microarchitecture, pages 338-349, Ann Arbor, MI, Nov. 29-Dec.1, 1995.

[3] R. Govindarajan, Erik R. Altman, and Guang R. Gao. Minimizing register requirements under resourceconstrained rate-optimal software pipelining. In Proc. of the 27th Ann. Intl. Symp. on Microarchitecture, pages 85-94, San Jose, CA, Nov. 30-Dec.2, 1994.

[4] P.Y-T. Hsu. Design of the R-8000 microprocessor. Technical report, MIPS Technologies Inc., Mountain View, CA, June 1994.

[5] Richard A. Huff. Lifetime-sensitive modulo scheduling. In Proc. of the ACM SIGPLAN '93 Conf. on Programming Language Design and Implementation, pages 258-267, Albuquerque, NM, June 23-25, 1993.

[6] Monica Lam. Software pipelining: An effective scheduling technique for VLIW machines. In Proc. of the SIGPLAN '88 Conf. on Programming Language Design and Implementation, pages 318-328, Atlanta, Georgia, June 22-24, 1988.

[7] Daniel M. Lavery and Wen mei W. Hwu. Unrollingbased optimizations for modulo scheduling. In Proc. of the 28th Ann. Intl. Symp. on Microarchitecture, pages 327-337, Ann Arbor, MI, Nov. 29-Dec.1, 1995.

[8] Josep Llosa, Mateo Valero, and Eduard Ayguade. Heuristics for register-constrained software pipelining. In Proceedings of the 29th Annual International Symposium on Microarchitecture, pages 250-261, Paris, France, December 2-4, 1996.

[9] Josep Llosa, Mateo Valero, Eduard Ayguade, and Antonio Gonzalez. Hypernode reduction modulo scheduling. In Proc. of the 28th Ann. Intl. Symp. on Microarchitecture, pages 350-360, Ann Arbor, MI, Nov. 29Dec.1, 1995.

[10] B. R. Rau and J. A. Fisher. Instruction-level parallel processing: History, overview and perspective. Jl. of Supercomputing, 7:9-50, May 1993.

[11] B. R. Rau and C. D. Glaeser. Some scheduling techniques and an easily schedulable horizontal architecture for high performance scientific computing. In Proc. of the 14th Ann. Microprogramming Workshop, pages 183-198, Chatham, MA, Oct. 12-15, 1981.

[12] B. R. Rau, M. Lee, P. P. Tirumalai, and M. S. Schlansker. Register allocation for software pipelined loops. In Proc. of the ACM SIGPLAN '92 Conf. on Programming Language Design and Implementation, pages 283-299, San Francisco, CA, June 17-19, 1992.

[13] B. Ramakrishna Rau. Iterative modulo scheduling: An algorithm for software pipelining loops. In Proc. of the 27th Ann. Intl. Symp. on Microarchitecture, pages 6374, San Jose, CA, Nov. 30-Dec.2, 1994.

[14] B. Ramakrishna Rau, David W. L. Yen, Wei Yen, and Ross A. Towle. The Cydra 5 departmental supercomputer - design philosophies, decisions, and trade-offs. Computer, 22(1):12-35, Jan. 1989.

[15] J. Wang and E. Eisenbeis. A new approach to software pipelining of complicated loops with branches. Research report no., Institut National de Recherche en Informatique et en Automatique (INRIA), Rocquencourt, France, Jan. 1993. 